Evaluating nurses knowledge and skills in the detection of child abuse in the Emergency

Department

Carolyn Keane, RN, BSc (Nursing) (for correspondence)

Staff Development Educator (Critical Care)

Fremantle Hospital

PO Box 480, Fremantle

W.A. 6959

Tel: (08) 93413224

Fax: (08) 94313531

Mobile: +61 0418197364

Email: carolyn.keane@health.wa.gov.au

Rose Chapman, RN, PhD candidate; MSc (Nursing)

Senior Lecturer

Director Clinical and Nursing Practice

School of Nursing and Midwifery

Curtin University of Technology

and

Nurse Research Consultant

Joondalup Health Campus

Tel: (08) 92662095

Fax: (08) 92662959

Mobile: +61 0401103460

Email: r.chapman@curtin.edu.au 


\title{
Evaluating Nurses Knowledge and Skills in the Detection of Child Abuse in the Emergency Department
}

\begin{abstract}
This commentary paper highlights the issue of child abuse and the important role that nursing staff working in the Emergency Department (ED) can play in halting the cycle of abuse. Child abuse is a worldwide problem that is occurring with increasing frequency. In fact, in Australia over the last five years the number of child protection referrals has more than doubled. As well as the immediate physical damage child abuse causes, it can also escalate to result in more serious injury and death. Furthermore, children who are abused in their early years of life are at increased risk of a range of adverse long-term developmental problems. Research has demonstrated that there is a significant lack of detection of suspected cases of child abuse in the ED. In fact the true incidence of children presenting to hospital EDs with abuse is difficult to determine, and many cases remain undetected. Nursing staff are perfectly positioned to detect the signs that a child may be at risk of abuse. However, in order to identify these signs it is essential that ED nurses have the knowledge and skills necessary to do so. Failure to consider the possibility of abuse will mean that the appropriate diagnosis is not made and the child is returned to an abusive environment. Therefore, this paper offers ED nurses recommendations for future directions in research and interventions to improve the detection of child abuse in Western Australia.
\end{abstract}

\section{KEYWORDS}

Child Abuse; Emergency Department; Intervention; Detection; Education 


\section{Introduction}

The motivation for this commentary paper stems from one of the authors personal involvement in a high profile child abuse case that occurred in the United Kingdom and the subsequent recognition of nurses lack of knowledge around the topic of child abuse when assessing paediatric patients in a South Metropolitan hospital Emergency Department (ED) in Western Australia (W.A). These experiences prompted the authors to reflect on current knowledge and research, specifically in the West Australian context, in the area of child abuse. A search of the literature failed to identify any West Australian research conducted to explore ED nurses knowledge or skills in identifying children at risk of abuse. Therefore, the aim of this paper is to discuss existing knowledge and research on child abuse and to make recommendations for future research and educational strategies which will lead to improved practice within the ED.

\section{Background}

Childhood is a broad term usually applied to the phase of development in humans between infancy and adulthood (Cole, et al., 2005). Childhood is a time in which vast physiological development and extensive learning about the environment occurs (Cole et al., 2005). Any traumatic events experienced during childhood can have far reaching consequences that can continue to impact throughout life (Blair, et al., 2003). One of the major traumatic events for any child is the experience of child abuse Child abuse is a worldwide problem that is occurring with increasing frequency (Sanders \& Cobley, 2005). Child abuse was first identified and defined by Kempe, et al. in 1962 who termed the phrase “Battered Child Syndrome”. They identified the problem as being “a clinical condition in young children who have received serious physical abuse, generally from a parent or foster parent (Kempe et al., 1962, p. 105) and recognised that the problem was a major cause of death and disability in children 
(Kempe et al., 1962). In recent times this definition has been expanded to include physical, sexual or emotional abuse and neglect of any child by any person (Spencer, 2002).

In 2002, the Department of Health and Human Services in the United States of America revealed that approximately 896,000 American children were the victims of physical, sexual or emotional abuse or neglect (Mulryan, Cathers \& Fagin, 2004). In the United Kingdom, in 2003, there were more than 30,000 children on the National Child Protection Register (Joughin, 2003). In Australia the number of children reported to child protective services for being at risk of abuse has more than doubled over the last five years from 107,134 in 1999-2000 to 252,831 in 2004-5. In 2004-05 there were 3,206 referrals for possible child abuse made to the relevant authorities in W.A. (Australian Institute of Health and Welfare, 2006). Although these figures demonstrate the rising rates of child abuse within Australia, many cases are still remaining undetected and unreported (Sanders \& Cobley, 2005).

\section{Physical Abuse}

Detecting cases of child abuse can be challenging as it can often be extremely difficult for the clinician to differentiate between accidental injury and abuse (Kos \& Shwayder, 2006). Physical abuse, which is often the easiest form of abuse to detect, involves any acts of violence that may result in pain, injury, impairment or disease. Some of the signs that may be seen include bruising, fractures and burns (Spencer, 2002).

Bruises are the most common presenting sign of physical abuse, being present in $90 \%$ of cases (Hudson \& Kaplan, 2006). As bruising frequently occurs in childhood as children grow and play, the clinician is required to explore certain factors of the child's presentation in order to ascertain if the bruising is accidental or deliberate. For example, the clinician needs to assess the developmental age of the child, the location, and the pattern of the bruise. From a developmental age point of view, bruises seen in 
babies should always be viewed as suspicious. Babies should not suffer from bruising as it is expected that they are supervised at all times and are not physically able to manoeuvre themselves into a situation which would allow them to impact with something, causing bruising (Vandeven \& Newton, 2006). The location of the bruise is important as accidental bruises are commonly seen on the anterior aspects of the body and over bony prominences where there is little cushioning and subcutaneous fat. Bruises on the posterior surfaces of the body such as the backs of arms and legs may possibly be inflicted as they imply that something has impacted the child instead of the child running into something. The patterning of bruises is also important as finger marks, bite marks or instrument marks are obviously inflicted injuries (Spencer, 2002). Finally, as multiple bruises in various stages of healing can also be a sign of abuse it is important that the whole child be examined to look for older bruising and injuries.

Another common cutaneous sign of child abuse, present in any age group, is burns. Inflicted burns are an important manifestation of child abuse as they imply a degree of intent to cause pain by the perpetrator (Kibayashi \& Shojo, 2003). Inflicted burns that are seen are typically “stocking” or "glove” marked and circumferential around the limb. These burns leave definite demarcation lines and have an absence of splash marks as the limb has been forcibly held in water (Spencer, 2002). Inflicted burns may outline the shape of the object used to cause the burn such as cigarettes or curling irons. The most commonly observed burn patterns seen in abuse are cigarette burns, which are characteristically small, deep, circular and often clustered in a group (Kos \& Shwayder, 2006).

As with bruising and burns, fractures in very young children, particularly non mobile infants, are often the result of abuse (Jenny, et al., 1999). Any type of fracture may be seen in an abused child but there are several specific fractures that carry a high association with child abuse (Hudson \& Kaplan, 2006). Spiral fractures of long bones (particularly the humerus and femur) are commonly seen as the result of 
abuse and are usually caused by the limb being forcibly twisted (Hobbs, 2002). Metaphyseal plate injuries in bones are also highly specific for abuse and are regularly seen in shaken babies as the shearing forces involved during violent shaking damages the weaker metaphysis, resulting in corner chip fractures of long bones (Hudson \& Kaplan, 2006). Rib fractures may also occur when babies are shaken, as the positioning of the perpetrators hands compresses the babies' chest causing bilateral posterior rib fractures (Australian Institute of Health and Welfare, 2005). Another fracture seen in children that may indicate abuse is a fracture of the skull, which can result from a deliberate head injury (Spencer, 2002).

An association has long been recognised between head injuries and child abuse (Joughin, 2003). Although the precise mechanism of injury to the brain in abusive head trauma is still not entirely clear, it is known that the shearing forces involved with abuse cause axonal injury to the developing brain (Glaser, 2000). Axonal injury is one of the key pathological features of abusive head injury and accounts for the primary symptoms and the clinical course of the injured child (Glaser, 2000). The presenting symptoms can be variable depending on the severity of the injury and can include a loss of consciousness, apnoea and cerebral oedema (Hudson \& Kaplan, 2006). As well as axonal injury, abusive head injury can cause tearing of the cerebral bridging veins with bleeding into the subdural space, leading to a subdural haematoma and retinal haemorrhages (Glaser, 2000).

It is often not the physical injury alone that leads to the detection of child abuse. Most presentations also possess behavioural indicators that add validity to the suspicion of abuse (Spencer, 2002). For example, there may be a delay between the injury occurring and the presentation of the child, the explanation given may be inconsistent with the injury seen and the interactions between the child and the parents may be abnormal (Sidebotham \& Pearse, 1997). As well as injuries associated with physical 
abuse, children may attend health care agencies demonstrating signs of other forms of maltreatment such as sexual abuse, emotion abuse or neglect.

Insert Table 1 (see page 22).

\section{Sexual Abuse}

Sexual child abuse differs from physical abuse in that it involves an adult using a child for sexual gratification (Hudson \& Kaplan, 2006). Sexual abuse is any act which exposes a child to, or involves a child in sexual processes beyond their understanding or contrary to accepted community standards (Chaney, 2000). Girls are twice as likely to be the victim of sexual abuse than boys (Hobbs, 2002). Furthermore, threats of sexual abuse can be used to illicit fear and inflict emotional abuse on a child as well.

\section{Emotional Abuse}

Emotional abuse is when distorted and excessive parental attitudes and actions result in emotional and behavioural problems in children (Spencer, 2002). Emotional abuse can include intimidation, harassment and concerted attacks on a child's self-esteem. Consistently belittling, embarrassing, blaming or withholding love and affection from children also amount to emotional abuse (Stevenson, 1998). Emotional abuse often accompanies other types of abuse and can result in children demonstrating extremes in behaviour, learning difficulties and nervous habits (Mulryan, et al., 2004). Aspects of emotional abuse are also often seen in cases of neglect (Truman, 2004).

\section{Neglect}

Neglect is the most common form of abuse and occurs when a person fails to perform behaviour that is a reasonable obligation of their relationship to the child. This mistreatment includes failure to 
adequately provide for the physical, cognitive, emotional, developmental, material or social needs of the child (Truman, 2004). The links between neglect and other forms of child abuse such as emotional and physical abuse are very strong and neglect is often seen in conjunction with other types of abuse (Stevenson, 1998). Recognising child neglect has been found to be extremely difficult as defining the boundaries of what constitutes neglect can be complex (Truman, 2004). However, neglected children most commonly exhibit delayed development and growth in addition to varying degrees of malnutrition (Truman, 2004). Neglected children often present to health care agencies with frequent illnesses and young infants are often seen with 'failure to thrive', that is failure over time to grow according to accepted standards for height, weight and development (Stevenson, 1998).

\section{Risk Factors}

Child abuse and neglect has been found to occur in all economic levels, ethnic backgrounds and family situations (Nayda, 2004). However, there are several situations in which a child may be at a higher risk of abuse or neglect. These circumstances include children from single, young or low-income families, parents who were themselves abused as children and children that currently live in a home where domestic violence is occurring (Chaney, 2000). Children who are born with special needs such as prematurity, birth defects or disabilities are also at elevated risks of abuse due to the increased level of stress placed on the parents caring for them (Russell, et al., 2004). The vast majority of perpetrators of child abuse are parents (Spencer, 2002). The result of increased stress on parents may be an isolated incident of abuse, or a repetitive pattern that occurs over a long period of time (Spencer, 2002).

Repetitive patterns of abuse, often escalate to result in more serious injury and death (Mulryan et al., 2004). Children who are abused in their early years of life are also at increased risk of a range of adverse long-term developmental problems (Glaser, 2000). For example, adolescents abused as children have more drug, alcohol and behavioural problems, mental health disorders and lower 
academic achievement than non-abused children (Australian Institute of Health and Welfare, 2005). A history of childhood maltreatment is now recognised as a major predisposing factor for parents who abuse, continuing the cycle of violence (Glaser, 2000). Like adolescents, the impact of child abuse has lasting repercussions on the adult (Sanders \& Cobley, 2005). Links between physical abuse in childhood and adult criminal activity, have been identified including aggression, anti-social behaviour and even murder (Australian Institute of Health and Welfare, 2005). Therefore, in order to safeguard the child and offer support to the abuser it is essential that these cases of abuse be identified as early as possible.

\section{Legislation}

To help identify cases of child abuse as early as possible mandatory reporting is now in place in all Australian states and territories, with the exception of W.A. However, recently the Premier of Western Australia Alan Carpenter announced that later this year the W.A Government will introduce legislation to expand mandatory reporting in W.A (Ainsworth, 2002). For years, the government has argued that such a move would only clog the system with unsubstantiated reports and place vulnerable children at further risk, but under the proposed system, doctors, nurses, teachers and police will be required by law to report when they have evidence that child sex abuse has occurred or is occurring (ABC News Online, 2007).

Even though mandated reporting is not currently legislated in W.A., clinicians still have a professional ethic to uphold the welfare of children (Ainsworth, 2002). Medical and nursing staff are ethically bound to notify their suspicions of abuse whether they are legally bound or not (Spencer, 2002). Therefore, it is important that clinical staff are familiar with child protection legislation relevant to them as they can play a significant role in the health and welfare of abused children because of their frequent contact with them and their families (Ainsworth, 2002). 


\section{Reporting of Child Abuse}

However, the under-reporting of abuse by health care workers such as dentists, general practitioners and primary health care nurses is well documented. (Largerberg, 2001; Russell et al., 2004). This under-reporting of abuse is due to the highly emotive nature of abuse and the fact that the evidence is not always easy to interpret. Health care workers are also often reluctant about "pointing the finger” or upsetting parents in case the accusations prove to be unfounded (Sanders \& Cobley, 2005).

Russell et al. (2004) conducted a cross-sectional survey in the United Kingdom, to assess primary health professional's perceptions and ability to recognise and report physical child abuse in their practice. All nurse professionals, GPs and dentists in Northern Ireland were sent a two-page questionnaire designed to assess their ability to recognise and report physical abuse. Even though 58\% of respondents (251) stated that they had seen a suspected case of child abuse only $47 \%$ (201) said that they had reported these suspected cases to the authorities. Eighty-six per cent of doctors, 96\% of nurses and health visitors and 38\% of dentists said they knew the mechanism for reporting suspicions of child abuse. Although the results from this study are valuable, the study only examined physical child abuse and excluded other forms of abuse or neglect. The authors reported that the nurse's response rates were low, however, the number of respondents was not stated in the study. The results from this survey suggest a professional reluctance to engage in recognizing and reporting abuse. This disinclination to report could be reduced by multi-professional and inter-agency training, support for professionals in practice and higher educational programs at under-graduate and post-graduate levels for nursing, dentistry and medicine (Russell et al., 2004).

\section{Child Abuse in the Emergency Department}


This reluctance by health care workers to recognise and report abuse is also present in the ED (Jenny et al., 1999; McKinney, Lane, \& Hickey, 2004; Sidebotham \& Pearse, 1997). In a busy ED it is easy to overlook the possibility of abuse. Lack of privacy and confidentiality, junior doctor's lack of experience and the pressure of time, all contribute to the difficulty in detecting cases of abuse (Sanders \& Cobley, 2005). As hospital EDs are often the first place where injured children come into contact with health care services, the role of ED staff can be instrumental in both recognising and responding to situations that may be indicative of abuse (Sanders \& Cobley, 2005).

A study conducted in Ireland by McKinney et al. (2004) examined whether possible cases of physical child abuse (as identified using 5 risk factors) gave rise to any suspicions of abuse in medical and nursing staff in ED. Questionnaires were completed by the triage nurse and attending doctor for every child attending with an injury at the Sligo and Letterkenny General Hospitals in Ireland during a six month period. During this time frame a total of 830 questionnaires were completed. It is unclear from the study if every child under 16 presenting to the two EDs during the six month trial were captured. The results showed that 13 children presented during the six month period with two or more positive risk factors, indicating that they may have been at risk of abuse. Neither the nurses nor the doctors involved expressed concerns about any of the patients who were identified by the positive risk factors. The results demonstrated that children presenting with an injury, that had two or more positive risk indicators for abuse failed to raise concerns in attending emergency department staff. The authors concluded that EDs must have clear policies and protocols for recognising and handling suspected abuse. The results from this study may be affected by the fact that 270 of the total 830 questionnaires used for the study had only had the first section completed by the nursing staff and that if the medical staff had completed the second section in these cases the results may have changed (McKinney et al., 2004) . 
Similar findings were found in an Australian study conducted by Ziegler, et al. in 2005 who examined how doctors in a general ED recognised and documented indicators of child abuse in children less than three years of age with fractures. The study was conducted in Liverpool Hospital in Sydney, New South Wales, where the Emergency Department Information System (EDIS) was searched to obtain a list of all children aged up to three years with fractures who presented to the ED. A retrospective review was carried out of the files of all presentations over a two-year period from January 2001 to December 2002. Ninety-nine patients up to three years of age presented with fractures over a two-year period. In 78 cases (80\%) the documentation did not indicate that the diagnosis of abuse had been considered or excluded. In 26 (22\%) of cases the history recorded in the notes was too brief to allow assessment of whether the injury was consistent with the history. In 16 (22\%) of the 72 records with an adequate history, the history as recorded by the emergency doctor was inconsistent with the injury. In 12 of these cases the doctor recognised the inconsistency and reported the child to the Department of Community Services; however in the other four cases the doctor did not indicate in the notes acknowledgement that the history was inconsistent. The findings of this study further confirm that emergency doctors do not document or assess for all of the indicators of child abuse in a high-risk population and do not consider the diagnosis of abuse in the majority of cases. This study was limited in its retrospective nature, which meant that it was only able to assess history and examination based on documentation in the notes. The small number of children with proven abuse combined with the retrospective nature of the study means that the results need to be interpreted with caution (Ziegler, et al, 2005).

Although research has clearly demonstrated the lack of detection of abuse by clinicians in the ED, findings of studies conducted in the UK have shown that the introduction of an education program and use of a reminder checklist can improve detection rates of child abuse (Sidebotham \& Pearse, 1997; Benger \& Mc Cabe, 2001; Benger \& Pearse, 2002). In 2001 Benger and McCabe conducted a two part 
audit to determine the effect of introducing a routine reminder mechanism into the ED notes and an education program on the staff's awareness of child abuse. They sampled 100 consecutive pre-school children's notes that presented with a burn or scald to Gloucestershire Royal Hospital and retrospectively reviewed the medical and nursing notes to assess the thoroughness of documentation and if the possibility of child abuse had been considered. After conducting the initial audit a short reminder checklist was devised to be used in the notes of any pre-school child that presented with burns or scalds. In addition, a program of improved education concerning the recognition and management of child abuse was undertaken. The results of the initial audit demonstrated that only 6\% (6) of preschool children presenting with burns or scalds had abuse considered by the doctor seeing the child and no children were referred on for further assessment. After conducting staff education and introducing a reminder checklist to improve awareness and documentation of child abuse in the ED this figure rose significantly to 65\% (65) with 3\% (3) being referred on for further opinion. This study demonstrated that a programme of intervention combining education and the use of a reminder checklist improved both awareness and documentation of child abuse, as well as referral rates for further assessment. It is difficult from the results of the study to identify to what extent the improvement seen was attributable to the intervention of the checklist and education or to the study itself highlighting the issue within the department. The rotation of medical staff through the ED between the two audits may also have affected the results (Benger \& McCabe, 2001).

\section{Emergency Department Nurses and Child Abuse}

ED nurses are often the first members of staff to interact with patients and families whilst they are waiting to see the doctor (Powell, 1997). Nursing staff are perfectly positioned to detect the signs that a child may be at risk of abuse (Joughin, 2003). However, it is essential that ED nurses have the knowledge and skills necessary to so identify a child at risk (Sanders \& Cobley, 2005). Failure to consider the possibility of abuse will mean that the appropriate diagnosis is not made and the child is 
returned to an abusive environment (Joughin, 2003). As recognition of child abuse is an essential skill for emergency nurses, there have been numerous articles published about child abuse aimed at emergency nurses (Joughin, 2003; Mulryan et al., 2004; Spencer, 2002). Many of these resources are educational, informing nurses how to identify the signs and symptoms of abuse. However, only a small number of research studies have been conducted that examine ED nurses and their role in the area of child abuse (Fagan, 1998; Feng \& Levine, 2005; Nayda, 2004).

Fagan (1998), an ED nurse in the UK, conducted a qualitative study that examined the perception of ED nurses in relation to the role they undertake when a victim of child abuse presents to the ED. Data were collected using a multi-purpose research tool that could be completed at an interview or independently by respondents. A small purposive sample of 14 experienced nurses was chosen on the basis of their number of years in the department. The majority of nurses $(n=10)$ did not perceive themselves as being skilled, as they did not see cases of abuse often. Those that did perceive themselves as skilled $(n=4)$ had over seven years experience in ED nursing. The author concluded that all the nurses included in the study had a broad base of knowledge and skills related to child abuse, which increased with experience (Fagan, 1998). However, it is very difficult to comment on the trustworthiness of these results as the methodology and data analysis techniques utilised in this study have not been made clear. The results of this study are also contradicted by studies conducted by Nayda (2004) and Feng and Levine (2005).

Nayda (2004) conducted another qualitative study in South Australia involving nurses who worked either in the ED or a paediatric department (PD). The study gathered data from two South Australian hospitals, both serving large lower socioeconomic populations. There were two methods of data collection. The first involved examination of 950 hospital records of children admitted to the EDs and PDs to consider nurse's documentation in relation to abuse from the two hospitals. Secondly, 
purposive sampling was used to identify 11 ED and PD nurses (from one of the two hospitals), who had experience in communicating about abused children who were asked to verbally respond to two scenarios in a one-hour, semi-structured interview. Three significant themes that were identified from the data included “codes of communication”, “acts of resistance” and “systemic malpractice”. The ED nurses documentation from both hospitals and the interviews suggested that ED nurses frequently overlooked or discounted potential abuse and often failed to record their suspicions. Unfortunately, many nurses declined to be involved in the study as they found the topic too sensitive as a larger number of participants may have strengthened the findings (Nayda, 2004).

However, a significantly larger quantitative study conducted by Feng and Levine (2005), supported Nayda’s (2004) findings. Feng and Levine (2005) used a stratified quota sampling method to survey Taiwanese paediatric, psychiatric and ED nurses to assess their knowledge, attitudes and experiences in reporting child abuse. The Child Abuse Report Intention Scale (CARIS) questionnaire was developed from data collected from an initial qualitative survey of 18 nurses. A total of 1400 from 1617 questionnaires were completed and returned (return rate 88\%) from 39 accredited hospitals throughout Taiwan. The results found that most of the nurses (86\%) had never reported a child abuse case, $21 \%$ said they had failed to report a suspected case of abuse and $80 \%$ had never had any child abuse education. The study highlighted the nurse's lack of knowledge and understanding and provided insight into the educational needs of nurses related to child abuse. The findings from this study are very valuable as the study was large, across multiple sites and utilised a random sampling method. The construct validity of the CARIS tool was also well established and the coefficient alphas calculated for the subscales of the questionnaire were high, ranging from 0.62-0.91 (Feng \& Levine, 2005). 


\section{Discussion}

Child abuse is occurring with increased frequency and many of these victims are seen by clinicians in the ED. However, research has shown that many cases are remaining undetected, by health care workers, due to the lack of formalised education, policies and procedures (Russell et al., 2004). In particular, ED clinicians are not engaging in discussions about high-risk cases and have demonstrated a persistent lack of knowledge about the clinical and social risk factors of abuse in children (Sanders \& Cobley, 2005). Furthermore, there still appears to be a culture of underreporting of suspected cases of child abuse in EDs, which is largely due to the fact that a significant proportion of medical and nursing staff do not receive formal training in identifying potential indicators of child abuse (Russell et al., 2004).

However, studies conducted in the UK have demonstrated that the intervention of an educational program and a reminder checklist can overcome these problems and have a significant impact on the consideration and documentation of possible cases of child abuse (Benger \& McCabe, 2001; Benger \& Pearse, 2002). Child abuse can be detected successfully by clinical staff if they have the knowledge to correctly identify the signs of potential abuse and a checklist to remind them to assess all children for those potential signs (Sanders \& Cobley, 2005). These interventions are currently utilised in W.A's specialised paediatric hospital, but not at the general hospital ED's in the state.

The research conducted thus far has focussed mainly on medical staff and has overlooked the potential impact that nursing staff can have in the detection and reporting of child abuse (Jenny et al., 1999; McKinney, Lane, \& Hickey, 2004; Sidebotham \& Pearse, 1997). Out of the numerous articles published in the area, only three specifically focussed on examining nursing staff's awareness and detection of abuse of which only one was Australian (Fagan, 1998; Feng \& Levine, 2005; Nayda, 2004). Nurses are in a prime position to be able to detect the signs of abuse as they are involved with 
the initial triage and assessment of the child and have extended contact with the child and their family before and after they have been seen by the medical staff. For these reasons it is imperative that ED nurses are fully aware of the risk factors and indicators of abuse, which will assist them to be more alert to the possibility of abuse when assessing their paediatric patients.

Although, the Australian federal and state governments are actively tackling the problem of child abuse (especially sexual abuse) by intervening in rural communities (ABC News Online, 2007) and introducing new legislation, these measures seem to be reactionary and not well thought out. With the probable introduction of mandatory reporting in this state, medical and nursing staff will be required under law to report any cases of suspected sexual abuse (Ainsworth, 2002). However, the introduction of this legislation will have a minimal impact if these named health professionals do not have the relevant knowledge or skills to allow them to detect these cases.

Even though multiple studies have found a continued lack of detection of potential child abuse cases by ED staff (Jenny et al., 1999; McKinney, et al., 2004; Sidebotham \& Pearse, 1997), no studies have been conducted in W.A to assess if clinical practice in this state follows the international trend. It would be valuable to conduct a study within a general ED in W.A that sees paediatric patients to assess nursing staff's knowledge and skills in identifying children at risk of abuse. In this way it would be possible to gain an understanding of how many cases may be going undetected. It is essential that research of this kind be conducted in WA so that education programs and policies can be developed and tailored to meet the needs of the local population.

Currently a study is planned to be conducted in a South Metropolitan Health Service Hospital ED that services children to identify if a gap in child abuse knowledge and practice exists among nursing staff. A questionnaire designed to assess clinicians knowledge, skills and practice in dealing with children at 
risk of abuse will be distributed to all nursing staff working in the ED. After completion of the initial study an ongoing education program and a reminder checklist will be implemented in the ED. The education program will consist of the development of an ongoing in-service program and a self directed learning package (SDLP). It will be expected that all nursing staff within the department either attend an in-service session or complete the SDLP within a specified time-frame. In collaboration with ED management, and other relevant stakeholders a reminder checklist will be developed to be utilised by nursing staff at triage. This checklist will be developed to prompt nursing staff at triage to take a thorough history from children and parents presenting to the department with an injury and to assist the clinician to consider the possibility of abuse. After a period of six months the initial questionnaire will be redistributed to all staff to assess if an increase in knowledge and skills and an improvement in practice have occurred. Regular communication will also commence with the social work department and paediatric unit to assess if detection and referral rates of possible child abuse have increased within the ED. The current hospital policy on the identification and responses to child abuse will be reviewed to develop an ED specific policy that can be easily understood and followed by the ED nursing staff.

\section{Conclusion}

The concept of child abuse is not new and extensive research has been conducted over the years to aid in fully understanding the phenomena. In Australia the number of cases of child abuse has doubled over the last five years. The literature clearly outlines the far reaching detrimental repercussions that child abuse can have on an individual. It is widely accepted that the detection of child abuse can be difficult. However, it is essential that health care professionals, including nursing staff, have the relevant knowledge and skills to enable them to accurately detect children at risk of abuse. Therefore, education programs must be provided to ED nurses. Furthermore, research needs to be conducted in W.A to identify how many cases of child abuse are being missed by clinicians in the ED. The findings 
of this research can be used to improve systems, policies and procedures of identifying children at risk of abuse to ensure that we are doing all we can to protect the future of children in W.A. 


\section{References}

ABC News Online. (2007). Retrieved June 5, 2007, from http://www.abc.net.au/news/

Ainsworth. (2002). Mandatory reporting of child abuse and neglect: Does it really make a difference? Child and Family Social Work, 7, 57-63.

Australian Institute of Health and Welfare. (2005). A picture of Australia's children Canberra: Australia's Institute of Health and Welfare.

Australian Institute of Health and Welfare. (2006). Child protection Australia 2004-05 [No CWS 26]. Canberra: Australian Institute of Health and Welfare.

Benger, J. R., McCabe, S. E. (2001). Burns and scalds in pre-school children attending accident and emergency: Accident or abuse? Emergency Medicine Journal, 18, 172-174. Retrieved December 15, 2005, from OVID MEDLINE database.

Benger, J. R., Pearse, A. V. (2002). Simple intervention to improve detection of child abuse in emergency departments. British Medical Journal, 324, 780-782.

Blair, M., Stewart-Brown, S., Waterstone, T., Crowther, R. (2003). Child Public Health. Oxford University Press: UK.

Chaney, S. (2000). Child abuse: Clinical findings and management. Journal of the Academy of Nurse Practitioners, 12(11), 467-471.

Cole, M., Cole, S.R., Lightfoot, C. (2005). The development of children (5th ed.). USA: Worth Publishers.

Department of Health Government of Western Australia. (2004). Responding to child abuse, neglect and the impact of family and domestic violence: Department of Health, Government of Western Australia.

Fagan, D. (1998). Child abuse and neglect: The knowledge and practise of the A\&E nurse. Accident and Emergency Nursing, 6, 30-35.

Feng, J. Y., Levine, M. (2005). Factors associated with nurses intention to report child abuse: A national survey of Taiwanese nurse's. Child Abuse and Neglect, 29, 783-789.

Glaser, D. (2000). Child abuse and neglect and the brain: A review. Journal of Child Psychology and Psychiatry, 41(1), 97-116.

Hudson, M., Kaplan, R. (2006). Clinical responses to child abuse. Paediatric Clinics of North America, 53, 2739.

Jenny, C., Hymel, K. P., Ritzen, A., Reinhart, S. E., Hay, T. C. (1999). Analysis of missed cases of abusive head trauma. Journal of American Medical Association, 282(7), 621-629. Retrieved August 18, 2006, from OVID Medline database.

Joughin, V. (2003). Working together for child protection in A\&E. Emergency Nurse, 11(7), 30-37.

Kempe, C. H., Silverman, F. N., Steele, B. F. , Droegemuller Silver (1962). The battered-child syndrome. Journal of the American Medical Association, 181(1), 17-24. 
Kibayashi, K., Shojo, H. (2003). Patterned injuries in children who have suffered physical abuse. Paediatrics International, 45, 193-195. Retrieved November 21, 2006, from OVID Medline database.

Kos, L., Shwayder, T. (2006). Cutaneous manifestations of child abuse. Paediatric Dermatology, 23(4), 311320.

Largerberg, D. (2001). A descriptive study of Swedish child health nurses' awareness of abuse and neglect. Abuse and Neglect, 25, 1583-1601.

McKinney, A., Lane, G., Hickey, F. (2004). Detection of non-accidental injuries presenting at emergency departments. Emergency Medicine Journal, 21, 562-564. Retrieved November 27, 2005, from OVID Medline database.

Mulryan, K., Cathers, P., Fagin, A. (2004). How to recognize and respond to child abuse. Nursing2004, 34(10), 52-57.

Nayda, R. (2004). Registered nurses communication about abused children: Rules, responsibilities and resistance. Child Abuse Review, 13, 188-199.

Powell, C. (1997). Protecting children in the accident and emergency department. Accident and Emergency Nursing, 5, 76-80.

Russell, M., Lazenbatt, A., Freeman, R., Marcenes, W. (2004). Child physical abuse: Health professionals perceptions, diagnosis and response. British Journal of Community Nursing, 9(8), 332-338. Retrieved August 18, 2006, from OVID CINAHL database.

Sanders, T., Cobley, C. (2005). Identifying non-accidental injury in children presenting to A\&E departments: An overview of the literature. Accident and Emergency Nursing, 13, 130-136. Retrieved June 10, 2006, from ScienceDirect database.

Sidebotham, P. D., Pearse, A. V. (1997). Audit of child protection procedures in accident and emergency departments to identify children at risk of abuse. British Medical Journal, 315, 855-856. Retrieved November 27, 2005, from OVID Medline database.

Spencer, D. (2002). Paediatric trauma: When it is not an accident. Accident and Emergency Nursing, 10, 143148. Retrieved June 10, 2006, from OVID CINAHL database.

Stevenson, O. (1998). Neglected children: Issues and dilemmas. Oxford: Blackwell Science.

Truman, P. (2004). Problems in identifying cases of neglect. Nursing Standard, 18(29), 33-38.

Vandeven, A. M., Newton, A. W. (2006). Update on child physical abuse, sexual abuse and prevention. Current Opinion in Paediatrics, 18, 201-205. Retrieved July 12, 2007, from OVID Medline database.

Ziegler, D. S., Sammut, J., Piper, A. C. (2005). Assessment and follow-up of suspected child abuse in preschool children with fractures seen in a general hospital emergency department. Journal of Paediatric Healthcare, 41, 251-255. Retrieved March 14, 2007, from OVID MEDLINE database. 
Table 1. Key Indicators of Physical Child Abuse

\begin{tabular}{|c|c|}
\hline Type of Injury & Indicators of Abuse \\
\hline Bruising & $\begin{array}{l}\text { - } \quad \text { Bruising in non-mobile infants } \\
\text { - } \quad \text { Bruises to the posterior of the body } \\
\text { - } \quad \text { Bruises in the shape of objects } \\
\text { - } \quad \text { Finger / bite marks }\end{array}$ \\
\hline Burns & $\begin{array}{ll}\text { - } & \text { Stocking or glove marked } \\
\text { - } & \text { Circumferential around limb } \\
\text { - } & \text { Burns in the outline of an object } \\
\text { - } & \text { Cigarette burns }\end{array}$ \\
\hline Fractures & $\begin{array}{ll}\text { - } & \text { Fractures in non-mobile infants } \\
\text { - } & \text { Spiral fractures of long bones } \\
\text { - } & \text { Posterior rib fractures } \\
\text { - } & \text { Skull fractures }\end{array}$ \\
\hline Head Injuries & $\begin{array}{ll}\text { - } & \text { Retinal haemorrhages } \\
\text { - } & \text { Subdural haematomas } \\
\text { - } & \text { Diffuse axonal injury }\end{array}$ \\
\hline
\end{tabular}

